\title{
Thin Film Deposition by Langmuir Blodgett Technique for Gas Sensing Applications
}

\author{
Sanit Malik*, Chandra Charu Tripathi \\ Department of Electronics \& Communication Engineering, University Institute of Engineering \& Technology (UIET), Kurukshetra \\ University, Thanesar, India. \\ Email: *maliksanit7@gmail.com
}

Received May $10^{\text {th }}, 2013$; revised June $11^{\text {th }}, 2013$; accepted July $1^{\text {st }}, 2013$

Copyright (C) 2013 Sanit Malik, Chandra Charu Tripathi. This is an open access article distributed under the Creative Commons Attribution License, which permits unrestricted use, distribution, and reproduction in any medium, provided the original work is properly cited.

\begin{abstract}
There are various technologies like CVD. Radio Frequency sputtering, spin coating etc. present for thin film deposition for various applications and for gas sensors. In this review, special attention is focused on the thin film deposition for gas sensing applications by using Langmuir Blodgett method. Langmuir Blodgett method also discussed briefly. Modified technique of Langmuir-Blodgett like Langmuir Schaefer method is discussed and various examples of Langmuir Blodgett techniques for gas sensing for space applications are included. Future prospects of gas sensing thin film deposition by Langmuir Blodgett technique are explained.
\end{abstract}

Keywords: Thin Films; LB Method; Gas Sensor; Molecular Electronics; Nanostructures

\section{Introduction}

Molecular electronics emerges as an important technology for the 21 st century. The electronics industries are consuming organic materials in LCD, xerography, acoustic transducer \& in various sensors etc. Various efforts have been made towards the formation of nanostructure materials like nanorods, nanotubes and various nanoparticles. So by using LB method, new materials with improved properties can be prepared. LB method is used to obtain high quality films. LB method allows changing various parameters of the thin film like molecules nature, deposition pressure, and composition of sub phase and counter ions [1]. LB method provides the control over the thickness of the film as in this method a single layer of molecules is formed on the liquid (water) surface and then this layer is transferred to the substrates. So by repeating this many times thin layers can be deposited on the substrates with controlled molecular thickness. As various gas sensors also use thin films for the sensing purpose so as to improve their parameters like sensitivity and selectivity it is required to obtain thin films of gas sensing materials. So control of thickness of film is achieved by LB method. The defect free films can be obtained by some changes in LB method [1]. By using LB method a thin layer of nanometer scale can be deposited on almost any kind of solid substrate.

\footnotetext{
*Corresponding author.
}

\section{LB Technique}

To understand the Langmuir Blodgett technique one should know some basics about the LB thin film deposition process like surface tension, surfactants, insoluble monolayer's, surface pressure, the Langmuir balance, surface pressure area isotherm etc. [2]. There is an attractive force present between the molecules of a liquid which is known as cohesion it depends on the properties of substance. Inside the liquid the molecules are attracted towards each other so that the balance is maintained in all directions but on air/water interface molecule have more attraction towards the liquid than the air $[3,4]$. The polar liquids have high surface tension. To lower the surface tension the force of attraction should be decreased by any means like by using contaminants. The illustration of the molecules interaction is shown in Figure 1.

As for LB method the material to be deposited on the substrate should be insoluble in water.

There are mainly two types of molecules i.e. hydrophobic (water insoluble) and hydrophilic (water soluble). The hydrophobic part mainly contains hydrocarbon chains and the polar groups $\left(\mathrm{OH}^{-}, \mathrm{NH}_{3}^{+}\right.$etc. $)$are present in the hydrophobic part [5]. Figure 2 shows the components of an ampiphile and orientation of an amphiphilic adopted at interface. So the surface tension of 


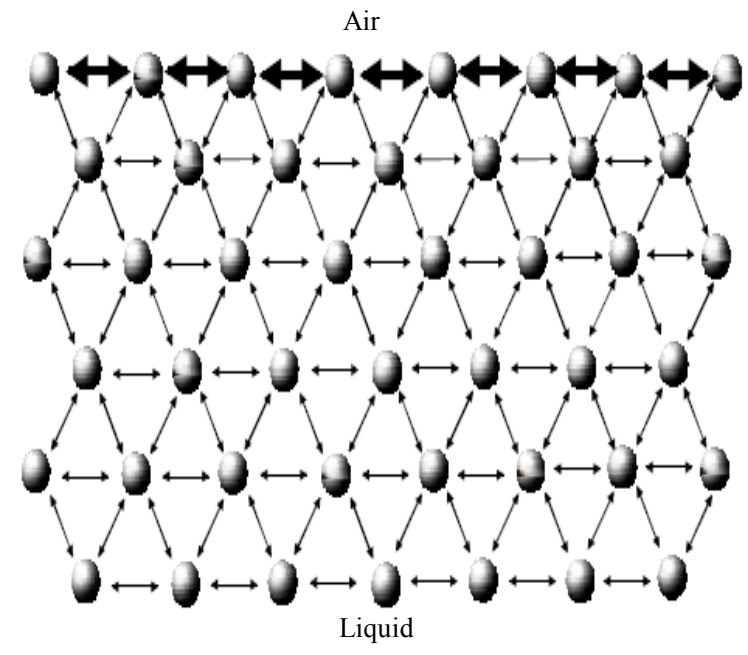

Figure 1. Molecule interaction in the air and water.

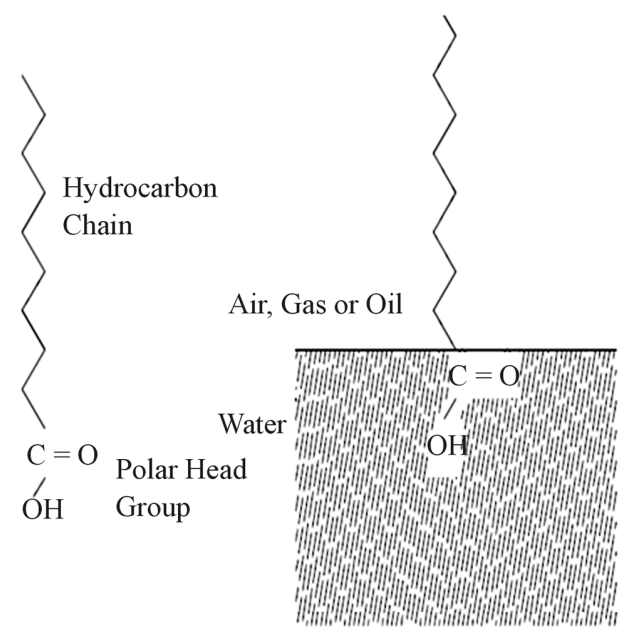

Figure 2. Components of an amphiphile and orientation of an amphiphile adopted at interface.

water is decreased when a surface active agent (surfactant) contacts with water [6,7]. A number of surface active agents are present with an amphiphilic nature that decreases the surface tension of the water. So the materials which are insoluble in water can be spread on the surface of water then a monolayer would be formed on the surface of the water.

The material on the water surface is such that the polar group is immersed in water and fluorocarbon chains are outside the water. Figure $\mathbf{3}$ shows the spread monolayer on the water surface.

The number of hydrocarbon group in the chain should be greater than 12 .

$\left(\mathrm{CH}_{2}\right)_{\mathrm{n}} \mathrm{n}>12$ but this chain should not be so long.

\subsection{Area Surface Pressure Isotherm}

The surface pressure as a function of the area of the water surface available to each molecule determines the properties of an amphiphilic material. Isotherm is mainly determined when the area between the barriers of LB is decreased i.e. by film compression at a constant rate with the surface pressure measurements by a computerized system [6-8]. As for the gas sensors, mainly metal oxides or fatty acids are used to form the thin film by LB technique so the isotherm for a typical fatty acid is shown in Figure 4.

From Figure 4, it is concluded that there are three different portions that fatty acids have i.e. gas $(\mathrm{G})$, liquid (L), and solid.

\subsection{Deposition of LB Film on Substrate}

There are two methods for thin film deposition by using LB technique i.e. vertical deposition and horizontal deposition [1]. In the horizontal deposition method two barriers on the Langmuir trough moves towards each other and the substrate is on the water so that the ampiphile present on the water surface deposits on the substrate as shown in Figure 5.

In the vertical deposition process of LB method the substrate is moved up and down on the water surface by a controlled computerized system [1]. In the vertical deposition process the substrate is moved up and down on the water surface by a controlled computerized system. Figure below shows the vertical deposition process. The

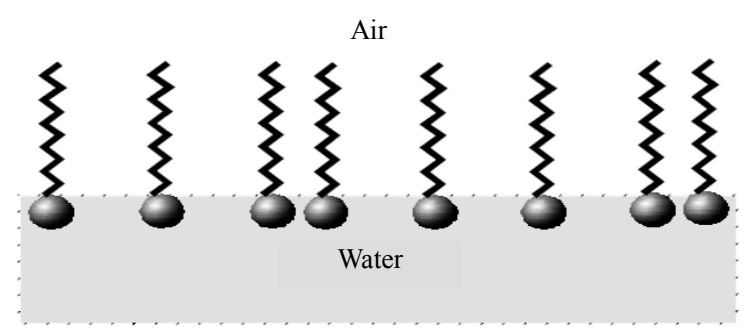

Figure 3. Spreaded monolayer on the water surface.

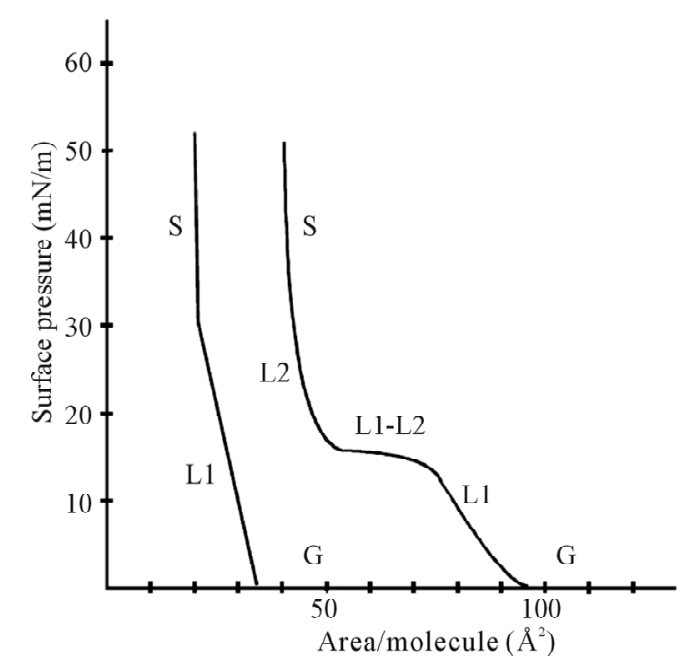

Figure 4. Typical isotherm of a fatty acid (left line). 
monolayers are deposited on the solid substrate. Different type of deposited LB films is present as shown in Figure 6 below.

When the monolayer's are deposited on the solid substrate then the surface pressure should be high enough to ensure that there is sufficient attractive force (cohesion) in the monolayer. So the nature of monolayer affects this surface pressure. The amphiphiles at surface pressure lower than $10 \mathrm{mN} / \mathrm{m}$ is sufficient to deposit the monolayer on the substrate.

\subsection{Langmuir Blodgett Technique for Gas Sensor}

The detection of various chemicals or gases is very important for industrial purposes, environment and for various applications. A number of gas sensing materials are present for the gas sensors like polymers, semiconductors, and metal oxides etc. [9]. The solid state sensors provide good sensing results amongst these as they have low cost, small size. So to enhance the sensing properties the molecular structure of the sensing material is very important. The LB method provides a control over the molecular structure of the films deposited on substrates. So by using Langmuir Blodgett technique a sensing thin layer can be deposited on the substrate. As the layer is thin then the sensor sensitivity will be improved because as the layer size decreases the sensitivity increases.

The gas sensors for space applications are the interested area of research these days. A number of organic compounds for sensing materials of gas sensors are used in many researches because of their molecular architecture [10]. The reactions which occur on the sensing material when a gas comes into the contact of the sensing material are responsible for the conductivity and resisti-

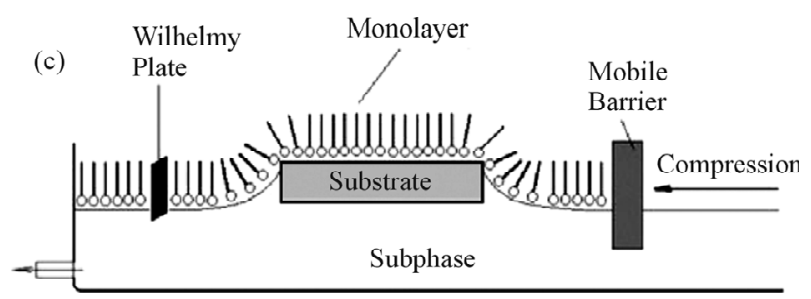

Figure 5. Horizontal depositions by LB method.

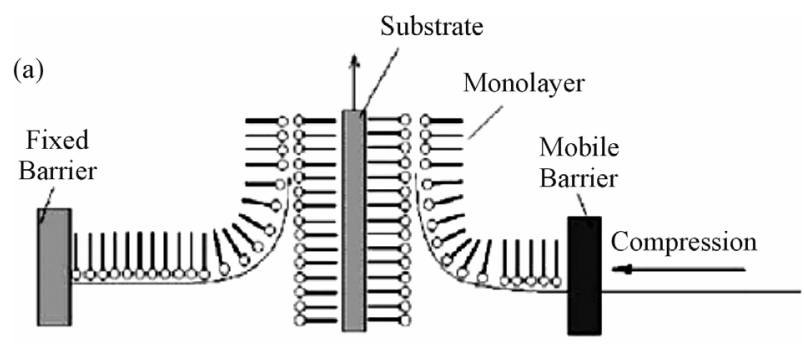

Figure 6. Vertical deposition of LB film. vity changes. The metal oxides conductivity is greatly affected by the volume or the surface defects on the thin layer of gas sensing materials. The resistance of thin film by LB method changes when the number of layers deposited is increased or decreased. Thinner the monolayer or film deposited on the substrate the sensor shows higher sensitivity and the response time of the sensor becomes short or less. [11] e.g. the response time of polyaniline films is less than that of the polyaniline and acetic acid mixed films because the thickness of the film affects the adsorption process [11]. i.e. the molecules of gases (like $\mathrm{NO}_{2}$ ) adsorbs easily on the thin film surfaces. Doping also inc. the sensor response or properties. The main problem of various solid state sensors is the sensitivity at room temperature so to improve this number of attempts have been made by the researchers. Some of them use the thermal decomposition of the thin films at $6000 \mathrm{c}$ for $2 \mathrm{~h}$ or more [12]. So that the thin layer can be deposited properly of the specific material and to remove the disadvantage of operating gas sensors at high temperature. Various efforts towards thermal decomposition have been made. The octadecylamine-stannate complexes with sodium stannate solution show thin layer formation by LB method and then after thermal decomposition this layer shows high sensitivity at room temperature [12]. Figure 7 as reproduced below shows the current vs voltage plot on a Quartz/ $\mathrm{SnO}_{2}$ structure.

\subsection{Factors Influencing the Sensitivity}

The operating characteristics of the gas sensors mainly depend on the surface reactions of the sensing material because the surface reactions of the material are responsible for the sensitivity. so the sensor sensitivity can be increased by using materials with very small grains sixes, and this simulated result agrees well with the experimental observation. Lu et al. [13] have indicated that the $\mathrm{SnO}_{2}$-based sensor response to $500 \mathrm{ppm} \mathrm{CO}$ increases drastically if the particle diameter becomes smaller than

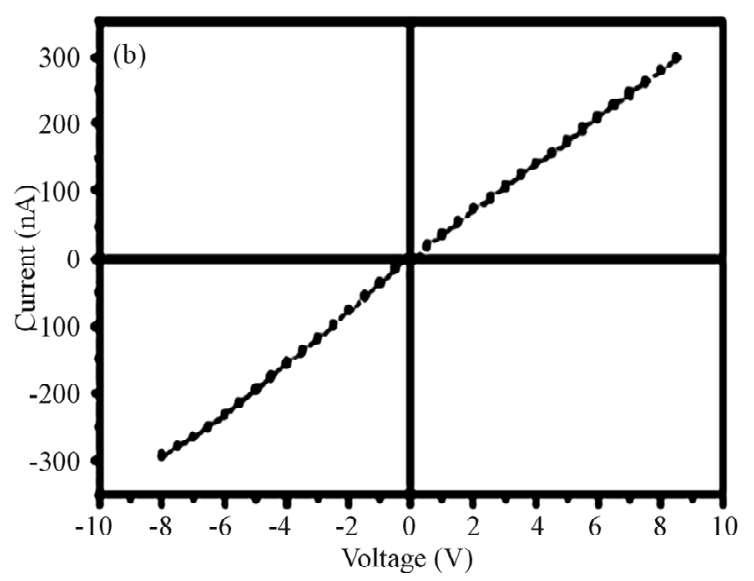

Figure 7. Current vs. voltage plot on quartz/ $\mathrm{SnO}_{2} /$ structure. 
$10 \mathrm{~nm}$ as shown in Figure 8.

The semiconductor sensing properties depends on reactions between the sensing material and the gases in the atmosphere, which cause a change in the resistance of the semiconductor due to charge transfer between the adsorbate and the adsorbent [14]. In the case of $\mathrm{NO}_{2}$, there is evidence of reactions directly with the semiconductor surface rather than with the oxygen chemisorbed at surface which is very useful for space applications.

The adsorbates originating from $\mathrm{NO}_{2}$ are essentially the same as those for $\mathrm{NO}$, since $\mathrm{NO}_{2}$ molecule dissociate easily over the tin oxide surface. These adsorbates can be factorised into three different types: two nytrosil types, $\mathrm{Sn}-\mathrm{NO}+$ and $\mathrm{Sn}-\mathrm{NO}-$, and the nitrite type $\mathrm{Sn}-\mathrm{O}-\mathrm{N}=\mathrm{O}$ [9]. The nitrite type does not play a role in gas sensing since it is not involved in any electron exchange with the bulk of the semiconductor. These results are in agreement with the work of Solymosi and Kiss [14] who proposed the following reactions:

$$
\begin{gathered}
\mathrm{NOg}+\mathrm{e}-\rightarrow \mathrm{NO}- \\
\mathrm{NOg} \rightarrow \mathrm{NO}++\mathrm{e}-
\end{gathered}
$$

where the second type of absorption is much weaker than the first one.

The temperature also affects the reactions of the gas sensors. C. Baratto, E. Comini, G. Faglia, G. Sberveglieri, M. Zhab, A. Zappettini discover the role of working temperature up to $400^{\circ} \mathrm{C}$.

The relative response is maximum (Figure 9) at relatively low temperatures $\left(200^{\circ} \mathrm{C}\right)$, due to the peculiar reaction mechanism of $\mathrm{NO}_{2}$ with $\mathrm{MOX}$ that does not involve oxygen chemisorbed species [14].

Nanostructure materials like carbon nanotubes and metal oxide nanowires provides superior performance over conventional approaches of gas sensing, due to their ability to direct selective uptake of gas species based on

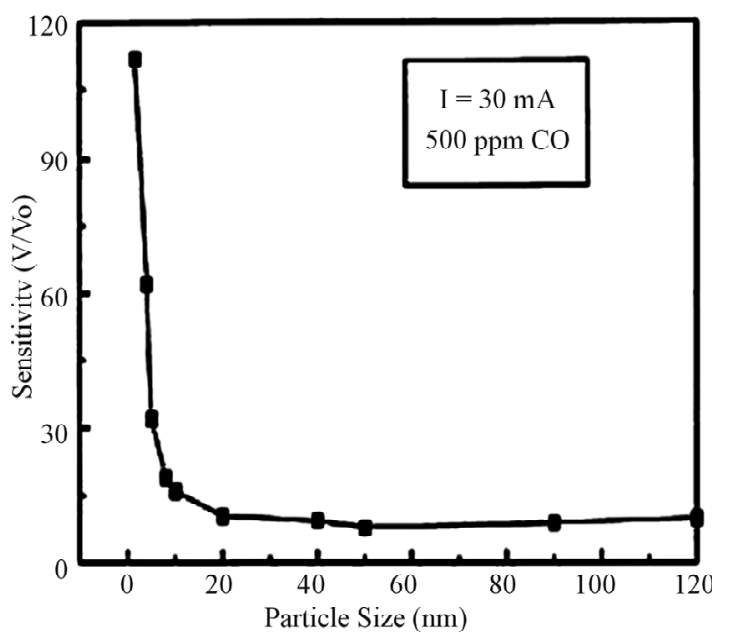

Figure 8. Effect of particle size on gas sensitivity for CO (adapted from [13]).

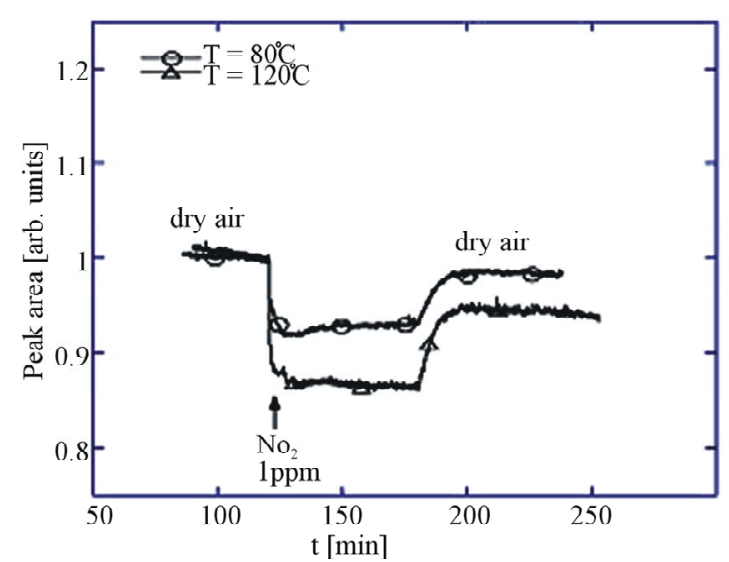

Figure 9. Kinetic response (normalized peak area intensity) toward $1 \mathrm{ppm}$ of $\mathrm{NO}_{2}$ at 80 and $120^{\circ} \mathrm{C}$ in dry air. (Adopted from [14]).

their pore size and chemical property. Nanostructure material has increased adsorptive capacity due to their large surface area [15]. CNTs can be doped with catalysts or mixed with polymers to achieve high selectivity. Various developments have been made for gas sensor which proves that CNT sensors offers very high sensitivity for the gases like $\mathrm{NO}_{2}$, ammonia, methane, acetone, benzene and toluene detection, with range in the lower ppm to $\mathrm{ppb}$ level and response time in few seconds to minutes [15].

LB technique is used to improve the gas sensor sensitivity when a thin film is deposited on a high density of acoustic energy substrate [9]. Various hydrogen gas sensors are designed using Langmuir Blodgett technique of thin film deposition. Lang and Lando in their research work showed the importance of LB method for halogen gases. They deposited the films on silicon substrates with a heater their study of phthalocyanine $\mathrm{lb}$ films for gases like $\mathrm{Cl}_{2}, \mathrm{Br}_{2}$ and $\mathrm{I}_{2}$ at different temperatures [16]. The maximum sensitivities for $\mathrm{Cl}_{2}$ is at $1300 \mathrm{c}$ for $\mathrm{Br}_{2}$ is at $600 \mathrm{c}$ and for $I_{2}$ is at $200 \mathrm{c}$. they found that the conductivity changes arise from both surface and the bulk effects. So from their experiment and research it may be concluded that as the film thickness decreases the response increases, but slowly. Higher the temperature, larger diffusion coefficient, resulting improves sensor response [16].

Most of the gas sensing materials senses various gases and gives measurable relative change in resistivity. Therefore, for gas sensors it is important to have high sensitivity with high selectivity. So that other gases cannot interfere with the sensor response. Various techniques are used for this to achieve high selectivity. The alklyne linked conjugated porphyrin dimer is a very useful material to achieve high selectivity as shown by Tepore and senta in their research. They LB method to deposit thin films of alklyne linked conjugated porphyrin 
dimer on the substrates and found that the selectivity of alklyne linked conjugated porphyrin dimer when exposed to $\mathrm{NO}$ and $\mathrm{NO}_{2}$ is maximum as shown in Figure 10 [10].

So these types of materials are very important for research work as these materials can sense gases at low concentrations with high selectivity.

LB films of polymers like polyaniline [17] are studied by various researchers for gas sensing. N. E. Agbor and M. C. Patty studied the technique of surface Plasmon resonance for LB films of polyaniline. The polymer used by them is polyaniline (emeraldine base form) on the substrates of glass and metalized slides [18].

The effect of 1, 2, 3 layers of polyaniline is shown in Figure 11 below.

As shown in Figure 12 the overlapping layers increases the resonance angle and half width at half maximum and decreases the resonance depth.

The exposure of single layer of polyaniline to the gases like $\mathrm{NO}_{2}$ shows the changes/improvement in reflectivity and resonance angle. For $\mathrm{NO}_{2}$ the changes found were similar to those as for conductivity changes

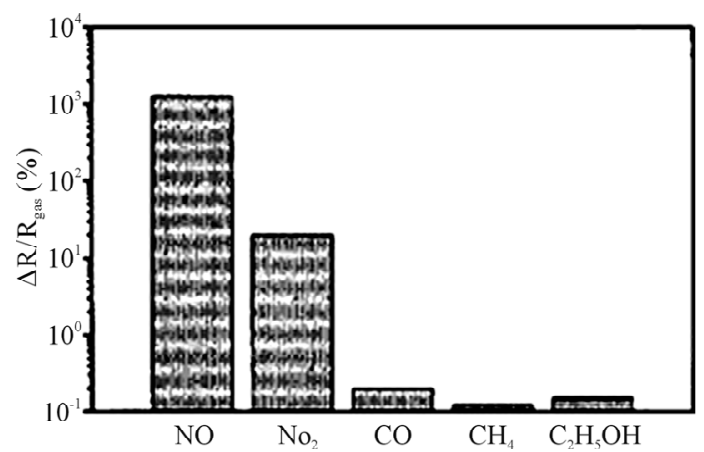

Figure 10. Relative resistance variations after exposure to various concentrations of gases.

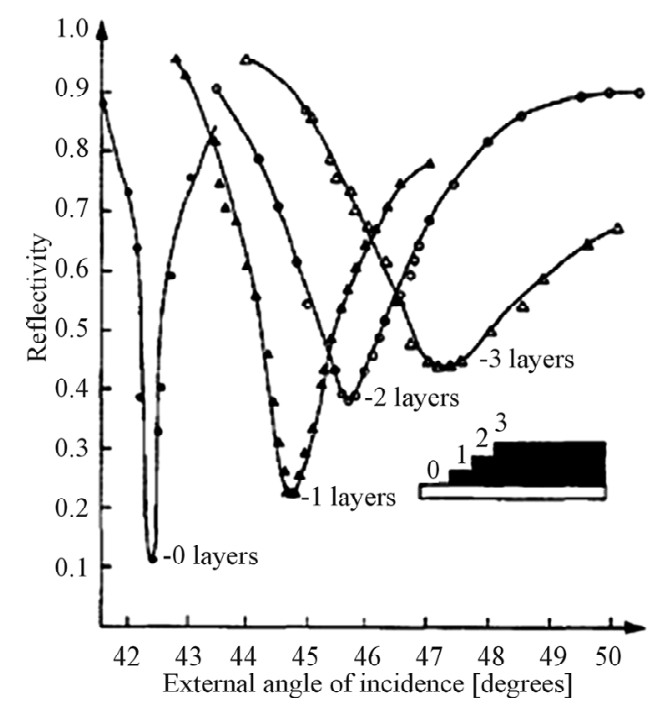

Figure 11. SPR curve of polyaniline LB films on a Ni/Ag substrate.
$[19,20]$. These polyaniline films are not of good quality as these films are not stable at the air water interface so to overcome this difficulty the polyaniline can be mixed with calix [10] resorciolarene derivative. So that good quality LB film can be obtained. The polyaniline and CA (calix [10] resorciolarene) mixture provides LB film of desirable nanoporous structure [21]. The CA is used because CA has a stable boat conformation as shown in Figure 13 below.

Which is responsible for the formation of polyaniline backbone in the hydrophilic cavity and another reason to select CA is that CA can show oxidation at ambient conditions also. The study of conductance changes of PA: CA LB films on exposure to $\mathrm{Hcl}$ and gases $\left(\mathrm{NH}_{3}\right)$ in air are made by N. V. Lavrinki, A. V. Nabok and are shown in Figure 14. The response of PA: CA films of 20 and 60 layers have been demonstrated in Figure 14.

The temperature also influences the response kinetics as shown by the research work of N. V. Lavrinki, A. V. Nabok.

So the PA: CA mixture provides stable monolayer films on the water surface. These films are insulating and become electrically conducting when these are doped with HCL. CA percentage decides the conductivity of these films. The relative conductivity of the as deposited

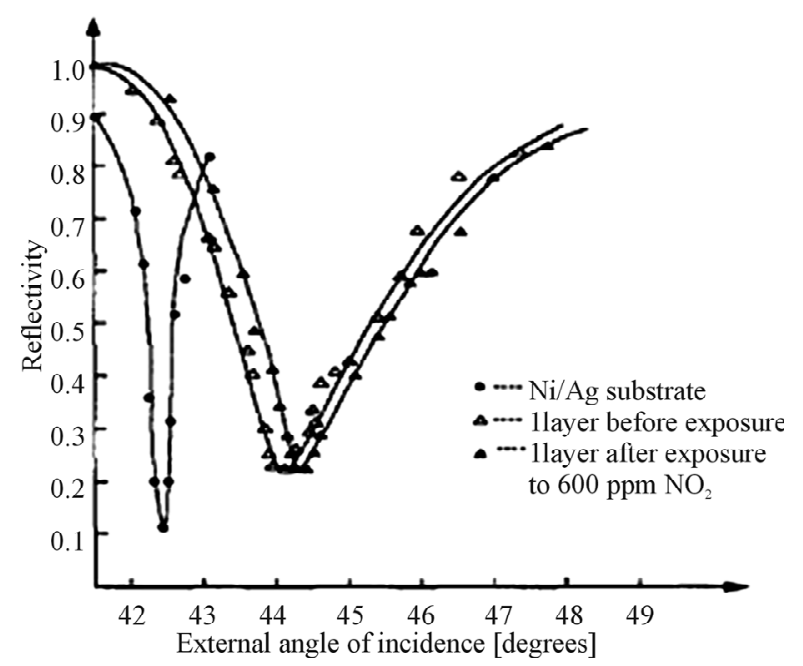

Figure 12. SPR curve of $\mathrm{NO}_{2}$ on a single layer of polyaniline.

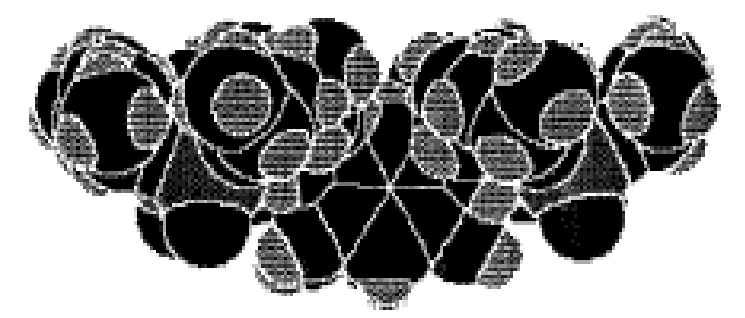

Figure 13. Boat conformation of calix [10] resorciolarene (CA). 
films vary with the temperature of measurement as shown in Figure 15. The films containing less than 20\% of CA act as pure polyaniline. The films which contain more than $20 \%$ of $\mathrm{CA}$ are more sensitive to gases and less to the moisture [21]. So from above research, it is clear that a lot of efforts have been made to improve the gas sensing properties of the gas sensor. But it needs more attention towards the structure of the thin films deposited by using LB technique. The nanoparticles which are of great importance can be mixed with various other organic materials as shown by T Di Luccio, F. Antolini, P. aversa. They mixed the carbon nanotubes and $\mathrm{CdBe}$ molecules and deposited the thin films by using LB technique [22]. They found that the layering properties of the films depend upon the surface pressure by which the single walled carbon nanotubes were deposited and also on the number of layer deposited. So it is clear that carbon nanotubes can be deposited on the substrates by using $\mathrm{Lb}$ technique and new organic nanoparticles

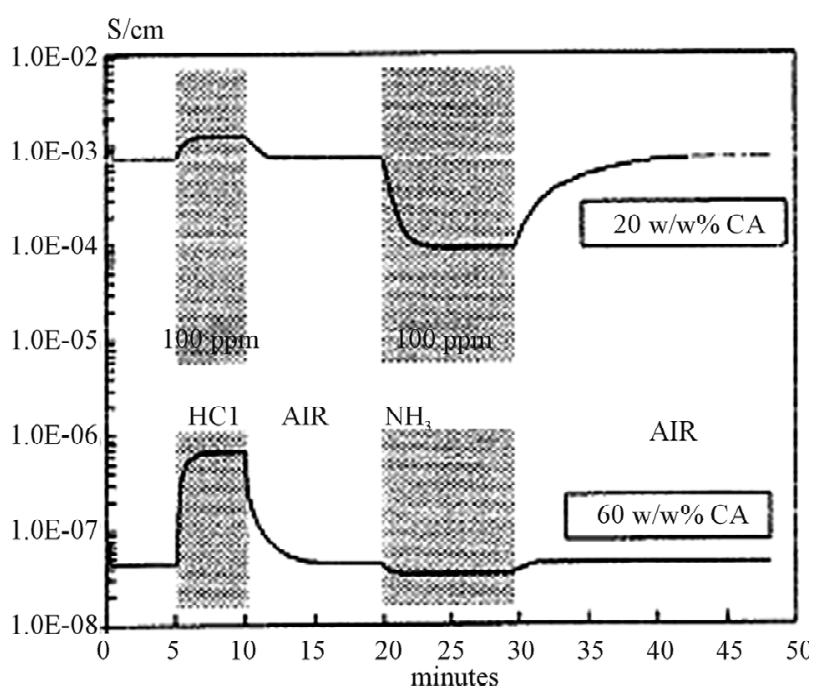

Figure 14. Responses of doped films with different CA percentage.

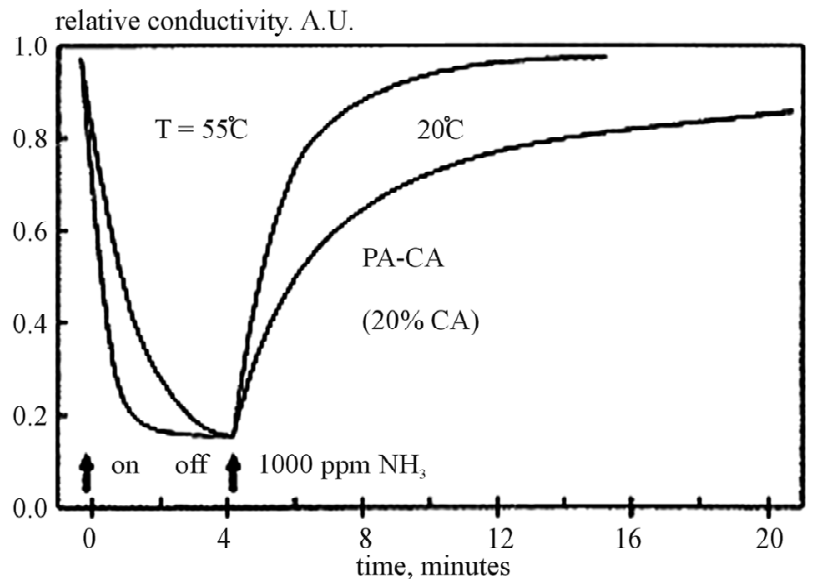

Figure 15. Influence of temperature on response kinetics. with improved responses can be designed for gas sensors.

As LB technique is very useful for thin film deposition but their films are not perfectly defect free and the stress and deformation problems can occur. So to avoid these problems some modified technique of LB method can be used such as Langmuir Schaefer method [23,24]. LS method is used to improve the deposition problems of LB films to obtain the films of high quality [25]. This method removes the problem which occurs during the vertical deposition process of the LB technique. If the impurities are available in the sub phase then the compound aggregation is very complex at the water air substrate so to overcome this problem LS method detach a part of the LB monolayer at the air water interface [1].

\section{Why Nanotechnology for Space}

The world-wide boom in nanotechnology funding and far reaching innovation pushed the space community to screen the applicable specific nanotechnology The NASA is established a Nanotechnology roadmap, reach up to 20 years in the future. Here are the positive arguments for Nanotechnology for space applications [26].

Advanced miniaturization is a gateway to the new world of science and technology.

Ultra small sensors, power sources, communication, navigation, and propulsion systems with very low mass, volume and power consumption are needed.

Nanotechnology provides a whole new technology to build device components and systems for entirely new space architectures.

- Networks of ultra small probes on planetary surfaces.

- Micro-rovers that drive, hop, fly, and burrow.

- Collection of micro spacecraft making a variety of measurements.

By the use of nanotechnology we can improve the sensitivity as various papers published on gas sensors focuses on the sensitivity of the sensors. There are some surface factors which can be used for improving the sensitivity of the sensors.

\section{Conclusion}

As various industries are using the organic materials and state of art of solid state engineering a lot of technical problems are present. That's why a lot of work is needed to be done for improving the quality of Langmuir Blodgett technique which can provide very high quality and defect and deformation free monolayer/lb films. The combination of molecular electronics and more complex nanostructure materials like nanotubes, nanorods, nanowires, nanobelts etc. can result in new organic materials with high quality and can be very useful for gas sensors. So by using the new techniques with LB methods, new superamolecular engineering methods can be developed 
which can be very promising for gas sensors, to improve gas sensors responses like high sensitivity, selectivity, stability and their operations at low temperature. So the Langmuir Blodgett technique of thin film deposition can be directed towards the development of very small size, low cost, gas sensors for space applications.

\section{REFERENCES}

[1] G. Zhavnerko and G. Marletta, "Developing LangmuirBlodgett strategies towards Practical Devices," Materials Science and Engineering B, Vol. 169, No. 1-3, 2010, pp. 43-48. doi:10.1016/i.mseb.2009.12.005

[2] Langmuir and Langmuir-Blodgett Films WHAT and HOW? http:/www.lot-oriel.com/files/downloads/ksv/en/langmui rblodgett en01.pdf

[3] A. W. Adamson, "Physical Chemistry of Surfaces," Wiley \& Sons, New York, 1976.

[4] D. J. Shaw, "Introduction to Colloid and Surface Chemistry," Butterworth \& Co., London, 1980.

[5] R. G. Laughlin, "The Aqueous Phase Behaviour of Surfactants," Academic Press Inc., San Diego, 1994.

[6] D. K. Chattoraj and K. S. Birdi, "Adsorption and the Gibbs Surface Excess,” Plenum Press, New York, 1984. doi:10.1007/978-1-4615-8333-2

[7] G. L. Gaines, "Insoluble Monolayers at the Liquid-Gas Interface,” Wiley-Interscience, New York, 1966.

[8] B. P. Binks, "Insoluble Monolayers of Weakly Ionising Low Molar Mass Materials and Their Deposition to Form Langmuir-Blodgett Multilayers," Advances in Colloid and Interface Science, Vol. 34, 1991, pp. 343-432. doi:10.1016/0001-8686(91)80053-M

[9] M. Penza, E. Milella and V. I. Anisimkin, "Gas Sensing Properties of Langmuir Blodgett Polyprrole Film Investigated by Surface Acoustic Waves," IEEE Transaction on Ultrasonics, Perroelectrics, and Frequency Control, Vol. 45, No. 5, 1998, pp. 1125-1132.

[10] A. Tepore, A. Serra, D. P. Arnold, D. Manno, G. Micocci, A. Genga and L. Valli, "Study of Gas Sensing Performance of Langmuir-Blodgett Films Containing an Alkyne Linked Conjugates Porphyrin Dimer."

[11] D. Xie, Y. D. Jiang, W. Pan, D. Li, Z. M. Wu and Y. R. $\mathrm{Li}$, "Fabrication and Characterization of Polyaniline Based Gas Sensor by Ultra-Thin Film Technology," Sensors and Actuators B, Vol. 81, No. 2-3, 2002, pp. 158-164.

[12] S. choudhary, C. A. Betty, K. G. Girja and S. K. Kulshreshtha, "Room Temperature Gas Sensitivity of Ultrathin $\mathrm{SnO}_{2}$ Films Prepared from Langmuir-Blodgett Film Precursors," Applied Physics Letters, Vol. 89, No. 7, 2006, 3 Pages. doi:10.1063/1.2336725

[13] F. Lu, Y. Liu, M. Dong and X. P. Wang, "Nanosized Tin Oxide as the Novel Material with Simultaneous Detection towards $\mathrm{CO}, \mathrm{H}_{2}$ and $\mathrm{CH}_{4}$," Sensors and Actuators $B$ : Chemical, Vol. 66, No. 1-3, 2000, pp. 225-227. doi:10.1016/S0925-4005(00)00371-3

[14] C. Baratto, E. Comini, G. Faglia, G. Sberveglieri, M. Zha and A. Zappettini, "Metal Oxide Nanocrystals for Gas Sensing," Sensors and Actuators B, Vol. 109, No. 1, 2005, pp. 2-6. doi:10.1016/j.snb.2005.03.091

[15] Carbon Nanotube Sensors for Gas Detection. www.nasa.gov/centers/ames/research/technology.../gas_d etection.html

[16] H.-Y. Wang and J. B. Lando, "Gas-Sensing Mechanism of PhthalocyanineLangmuir-Blodgett Films," Langmuir, Vol. 10, No. 3, 1994, pp. 790-796.

[17] A. J. Milton and A. P. Monkman, "A Comparative Study of Polyaniline Films Using Thermal Analysis and IR Spectroscopy," Journal of Physics D: Applied Physics, Vol. 26, No. 9, 1993, pp. 1468-1474. doi: $10.1088 / 0022-3727 / 26 / 9 / 020$

[18] N. E. Agbor, J. P. Cresswell, M. C. Petty and A. P. Monkman, "An Optical Gas Sensor Based on Polyaniline Langmuir-Blodgett Films," Sensors and Actuators B: Chemical, Vol. 41, No. 1-3, 1997, pp. 137-141. doi:10.1016/S0925-4005(97)80286-9

[19] N. E. Agbor, M. C. Petty, A. P. P. S. Vikus and J. R. Sambles, "Cobalt Phthalocyanine as a Basis for the Optical Sensing of Nitrogen Dioxide Using Surface Plasmon resonance," Thin Solid Films, Vol. 221, No. 1-2, 1992, pp. 311-317. doi:10.1016/0040-6090(92)90833-W

[20] D. G. Zhu, N. C. Petty and M. Harris, “An Optical Sensor for Nitrogendioxide Based on Copper Phthalocyanine Lanamuir-Blodgett Film," Sensors and Actuators B, Vol. 2, No. 4, 1990, pp. 265-269. doi:10.1016/0925-4005(90)80152-P

[21] N. V. Lavriky, D. De Rossiz, Z. I. Kazantsevay, A. V. Naboky, B. A. Nesterenkoy, S. A. Piletskyx, V. I. Kalchenkok, A. N. Shivaniukk and L. N. Markovskiyk, "Composite Polyaniline/Calixarene Langmuir-Blodgett Films for Gas Sensing," Nanotechnology, Vol. 7, No. 4, 1996, pp. 315-319. doi:10.1088/0957-4484/7/4/002

[22] T. Di Luccio, F. Antolini, P. Aversa, G. Scalia and L. Tapfer, "Structural and Morphologica Investigation of Langmuir-Blodgett SWCNT/Behenic Acid Multilayers". arxiv.org/pdf/cond-mat/0312395

[23] P. Bertoncello, A. Notargiacomo and C. Nicolini, "Langmuir-Schaefer Films of Nafion with Incorporated $\mathrm{TiO}_{2}$ Nanoparticles," Langmuir, Vol. 21, No. 1, 2005, pp. 172177. doi:10.1021/la0488371

[24] V. Ruiz, P. G. Nicholson, S. Jollands, P. A. Thomas, J. V. Macpherson, P. R. Unwin, "Molecular Ordering and 2D Conductivity in Ultrathin Poly(3-Hexylthiophene)/Gold Nanoparticle Composite Films," The Journal of Physical Chemistry B, Vol. 109, No. 41, 2005, pp. 19335-19344. doi:10.1021/jp053647k

[25] K. B. Blodgett and I. Langmuir, "Built-Up Films of Barium Stearate and Their Optical Properties," Physical Review, Vol. 51, No. 11, 1937, pp. 964-982. doi:10.1103/PhysRev.51.964

[26] Potential Applications of Micro and Nano Technology on Space Transportation System. www.silicondesigns.com/pdfs/spacepaper.PDF 